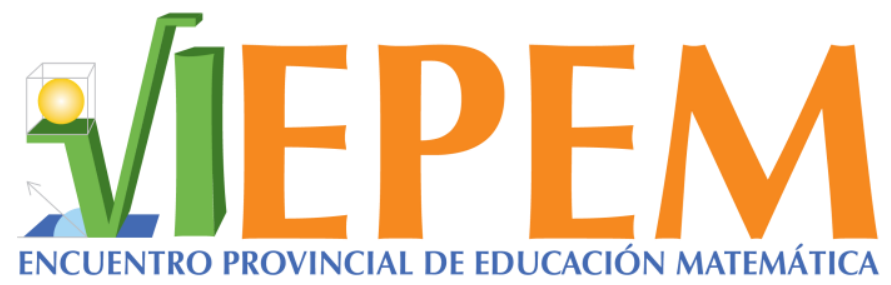

VI Encuentro Provincial de Educación Matemática.

27 al 29 de setiembre, 2017. Puntarenas, Costa Rica.

\title{
Sigan por ese camino hasta que se termine el pavimento: sugerencias prácticas para abordar geometría analítica desde la visión sociocultural de las matemáticas
}

\author{
Marcela García Borbón \\ marcela.garcia.borbon@una.cr \\ División de Educología-Universidad Nacional \\ Costa Rica
}

Jesennia Chavarría Vásquez

jessenia.chavarria.vasquez@una.cr

Escuela de Matemática

Universidad Nacional

Costa Rica

Margot Martínez Rodríguez

margot.martinez.rodriguez@una.cr

Escuela de Matemática-Universidad

Nacional

Costa Rica
María Elena Gavarrete Villaverde maria.gavarrette.villaverde@una.cr

Escuela de Matemática

Universidad Nacional

Costa Rica

Gerald Benavides Guido

gerald.benavides.guido@est.una.ac.cr

Estudiante

Escuela de Matemática

Universidad Nacional

Costa Rica

\section{Resumen}

Este taller describe una secuencia de actividades para la enseñanza de la Geometría Analítica en Educación Secundaria, implicando el uso de la visión sociocultural de las matemáticas, dado que se enmarca dentro del proyecto PPA-017615, titulado Formación Docente bajo la Visión Sociocultural de las Matemáticas, adscrito a la Escuela de Matemática de la Universidad Nacional.

Un rasgo de la idiosincrasia costarricense consiste en dar las direcciones postales de una forma descriptiva particular, muy arraigada en aspectos

Taller

García, M.; Chavarría, J.; Martínez, M.; Gavarrete, M. E. y Benavides, G. (2017). Sigan por ese camino hasta que se termine el pavimento: sugerencias prácticas para abordar geometría analítica desde la visión sociocultural de las matemáticas. En Y. Morales-López, M. Picado, R. Gamboa, C. Martínez, M. Castillo y R. Hidalgo (Eds.), Memorias del VI Encuentro Provincial de Educación Matemática, Costa Rica, 2017 (pp. 44-46). Heredia: Universidad Nacional. ISBN: 978-9968-96615-3. DOI: http://dx.doi.org/10.15359/epem.6.11 
Sigan por ese camino hasta que se termine el pavimento: sugerencias prácticas para abordar geometría analítica desde la visión sociocultural de las matemáticas

socioculturales, por lo que el eje central del taller es aprovechar esta característica tan propia de los ticos en el aula de matemática para la enseñanza de la Geometría Analítica. Se espera que los docentes participantes generen reflexiones sobre la forma en que estas actividades pueden ser integradas en la contextualización activa, que promueve los programas vigentes del Ministerio de Educación Pública (MEP, 2012).

Palabras clave: educación matemática; visión sociocultural; etnomatemática; geometría analítica; contextualización activa.

\section{Introducción}

Los programas vigentes de matemática del Ministerio de Educación Pública de Costa Rica (MEP, 2012) fueron concebidos acorde a las tendencias actuales en la Educación Matemática, por lo que brindan un significativo interés a la contextualización activa.

El taller que se presenta, es una adaptación para Educación Secundaria de otra taller concebido en el marco del curso de "Enculturación matemática y Etnomatemática" que se imparte a docentes de educación primaria, dentro del proyecto Formación Docente en la visión sociocultural de las matemáticas de la Escuela de Matemática de la Universidad Nacional de Costa Rica.

Los fundamentos teóricos de dicho proyecto, han sido desarrollados a partir de las ideas de Alan Bishop sobre la Enculturación Matemática como proceso que enriquece la formación docente (Bishop, 1999); así como los aportes del Programa de Etnomatemática, cuyo principal precursor es Ubiratán D'Ambrosio y que promueven la innovación pedagógica a partir de resultados de investigación en la visión sociocultural de las matemáticas, que ejerza impacto social en las comunidades, propicie la justicia social y facilite el ejercicio de una ciudadanía plena (D’Ambrosio, 2008).

Este taller se enfoca sobre dos de las áreas de conocimiento establecidas por el MEP en los programas de estudio, Geometría y Medidas; donde el propósito central, es abordar los temas de localización y ubicación espacial desde la visión sociocultural de las matemáticas para enriquecer el aprendizaje de la geometría analítica con actividades contextualizadas en el entorno del estudiante, utilizando uno de los rasgos que más nos dotan de identidad a los costarricenses que es la forma peculiar en la que describimos oralmente las direcciones.

\section{Metodología del taller}

La metodología que se utilizará en el taller abarca tres actividades. En primer lugar, se describe y analiza la dirección postal de los participantes del taller, reflexionando sobre los rasgos identitarios y los aspectos matemáticos implícitos en estas direcciones. 
Sigan por ese camino hasta que se termine el pavimento: sugerencias prácticas para abordar geometría analítica desde la visión sociocultural de las matemáticas

Como segunda actividad, se presenta una imagen satelital de una ciudad urbana costarricense, donde se identifiquen claramente las calles y avenidas, de modo que puedan implicarse actividades relacionadas con el plano cartesiano, tales como: distancia entre puntos, ecuación de una recta, radio de una circunferencia, entre otros. La tercera actividad utiliza una imagen satelital de una ciudad rural costarricense para la resolución de problemas contextualizados a través de la geometría analítica.

El cierre del taller involucra la socialización de los participantes sobre la experiencia y sobre la viabilidad de implementar una actividad similar en su aula.

\section{Resultados esperados}

Este taller cuenta con una experiencia piloto desarrollada con estudiantes de la Licenciatura en Enseñanza de la Matemática, por lo que se puede asumir que algunos resultados esperados son los siguientes:

- Manifiestan que es viable encontrar una serie de elementos matemáticos en la descripción de las direcciones postales en Costa Rica, tales como: paralelismo, perpendicularidad, distancia, escalas, entre otros.

- Manifiestan que es viable replicar el taller en aulas de secundaria.

- Manifiestan que genera interés por participar en el aprendizaje y se constata la utilidad de la geometría analítica en el contexto.

El abordaje de temáticas afines a la localización o ubicación espacial desde la perspectiva que se propone en este taller, es erradicar modelos tradicionales de enseñanza ajenos a la realidad del estudiante y potenciar estrategias, desde la clase de matemática, que propicien un cambio social, fortaleciendo la identidad de las comunidades.

\section{Referencias}

Bishop, A. J. (1999). Enculturación Matemática. Barcelona: Paidós.

D'Ambrosio, U. (2008). Etnomatemática - Eslabón entre las tradiciones y la modernidad. México: Limusa.

Ministerio de Educación Pública (2012). Programas de Estudio de Matemáticas, Reforma Curricular en Ética, Estética y Ciudadanía. San José: Ministerio de Educación Pública, Republica de Costa Rica.

\section{(2) $\odot \Theta \Theta$}

Esta obra está bajo una licencia de Creative Commons Reconocimiento-NoComercialSinObraDerivada 4.0 Internacional. 